\title{
Scarifier Device
}

National Cancer Institute

\section{Source}

National Cancer Institute. Scarifier Device. NCI Thesaurus. Code C149890.

Device for scratching the skin. 